\title{
Non-linear Distortion Cancellation and Symbol-based Equalization in Satellite Forward Links
}

\author{
Svilen Dimitrov
}

\begin{abstract}
In this paper, a low-complexity symbol-based equalizer that performs non-linear distortion cancellation is proposed for application at the user terminal in the DVB-S2X satellite forward link. The channel is comprehensively modelled, including the non-linear travelling wave tube amplifier (TWTA) characteristics, the input-multiplexing (IMUX) and output-multiplexing (OMUX) filter responses at the satellite transponder, and the phase noise at the user terminal, according to the very-small aperture terminal (VSAT) reference scenario. Two detectors in the cancellation loop are considered, comparing the packeterror rate (PER) performance of simple maximum likelihood (ML) demodulation with hard decision to soft information exchange with low-density parity-check (LDPC) decoder, and showing only marginal improvement with the latter solution. The PER performance is compared against a number of predistortion techniques at the transmitter, such as dynamic data pre-distortion, successive data pre-distortion, and static data predistortion. The novel receiver demonstrates superior performance even with one iteration of distortion cancellation, while the joint application of successive data pre-distortion and iterative symbol-based equalization shows up to 4.95 -dB energy efficiency gain for 32-level amplitude and phase-shift keying (32-APSK). The computational complexity is also evaluated. The improved receiver is particularly suitable for application with higher order modulation, a wide-band carrier, and low roll-off factors.
\end{abstract}

Index Terms-Satellite communication, non-linear distortion, equalization, pre-distortion, energy efficiency.

\section{INTRODUCTION}

The recently published extension of the second-generation digital video broadcasting system for satellite broadcasting and unicasting (DVB-S2X) standard [1] introduces a number of improvements for the air interface of the satellite forward link. Key advances include: 1) wide-band operation with transponder bandwidths of $225 \mathrm{MHz}$ for application with Ka-band transponders, 2) higher-order amplitude and phase-shift keying (APSK) modulation up to 256-APSK and additional lowdensity parity-check (LDPC) codes for more efficient resource utilization with finer signal-to-noise ratio (SNR) threshold granularity, and 3) lower roll-off factors down to $5 \%$ in order to reduce the bandwidth occupied by a carrier. Targeting applications in broadcast or unicast broadband scenarios for highthroughput-satellite (HTS) systems [2], these advances enable the shift towards more bandwidth-demanding applications and services, the adaptation to traffic demand across the coverage area, and the decrease of the cost per transmitted bit.

This work was presented in part at the 8th Advanced Satellite Multimedia Systems Conference (ASMS 2016), Palma de Mallorca, Spain.

S.Dimitrov is with the German Aerospace Center (DLR), Satellite Networks Department, 82234 Wessling, Germany (e-mail: svilen.dimitrov@dlr.de).
Transmission over the satellite forward-link channel suffers from linear and non-linear distortions. In order to maximize the spectral efficiency, state-of-the-art satellite communication systems resort to single-carrier utilization of the satellite transponder bandwidth, using a very low roll-off factor and very high symbol baud rates [3], e.g., for $4 \mathrm{~K}$ television broadcasting in the direct-to-home (DTH) scenario or broadband interactive Internet protocol (IP) services in the verysmall-aperture terminal (VSAT) scenario. HTS links with a wide-band single-carrier transponder are also applied to cover emerging markets for mobile platforms, such as broadband connectivity on airplanes, cruise ships and trains, as well as professional applications. However, due to imperfect magnitude and group-delay responses of the input-multiplexing (IMUX) and output-multiplexing (OMUX) filters on board the satellite, linear distortion is introduced in the form of intersymbol interference (ISI). In addition, due to the power limitation of the satellite, the travelling wave tube amplifier (TWTA) is operated close to saturation. Because of the non-linear transfer characteristic of the TWTA, non-linear distortion, in the form of constellation warping and clustering at the receiver demodulator, is introduced when waveforms with high peakto-average-power ratio (PAPR) are used for transmission, e.g., pules-shaped time-division multiplexing (TDM) employing constellations up to 256-APSK with multiple amplitude rings. Without adequate channel compensation, these adverse effects can reduce the energy efficiency and spectral efficiency gains expected with the application of tighter roll-off factors, higher symbol rates, and higher modulation orders.

In state-of-the-art satellite communications, the non-linear distortion can be handled by means of output back-off (OBO) [4] adjustment. This approach is known to penalize the energy efficiency of the system when high OBO is applied. Advanced non-linear compensation techniques include a number of pre-distortion techniques at the transmitter, such as data pre-distortion [4], [5], [6], [7], [8] or signal pre-distortion [5], [9], [10]. Static data predistortion [3], [4], [5], [11] is the state-of-the-art solution in DVB-S2X. A common approach to handle the ISI is equalization [5], where knowledge of the channel, obtained for instance by channel estimation, is used to minimize the ISI. A linear equalizer has been introduced in the DVB-S2X receiver [3].

Decision-directed advanced equalization techniques [12] applied at the receiver are known to provide very good performance in channels with memory. A turbo equalizer 
has been proposed for application over non-linear satellite channels in [13]. Using soft information exchange with the LDPC decoder and a channel model based on Volterra series, this equalizer computes minimum mean-squared error (MMSE) filters for the non-linear terms and performs distortion cancellation in an iterative fashion. A novel symbolbased iterative equalization scheme introduced in [14] reduces the complexity of the receiver by using maximum likelihood (ML) demodulation with hard decision in the cancellation loop and a simplified model for the received symbols at the input of the demodulator. Using a buffered physical layer (PL) frame, a first-order decomposition is applied to the received symbols, based on symbol-based constellation scaling factors representing the warping effects plus a non-linear noise component containing the higher non-linear orders. This decomposition can be used to reconstruct and cancel the interfering component, using channel models based on measured responses of the deterministic distortion sources along the chain, such as IMUX/OMUX responses and TWTA transfer characteristics. In addition to non-linear distortion, memory effects in the form of ISI are also compensated due to the fact that the equalizer operates on a large block of consecutive symbols. A related successive data pre-distortion technique at the transmitter has been proposed in [8] which also employs the measured responses of the IMUX/OMUX filter and TWTA characteristics to estimate and cancel the interference. The performance of the symbol-based equalizer with non-linear distortion cancellation has been evaluated in [14], [15] in satellite return links with time-division multiple access (TDMA) for a modulation setup according to the digital video broadcasting return channel via satellite 2 (DVB-RCS2) standard [16], and demonstrates significant gains in energy efficiency, while maintaining low implementation complexity.

In this paper, symbol-based equalization with non-linear distortion cancellation is proposed for the satellite forward link as an addition to the standard linear equalizer at the receiver. As compared to [14], the equalizer has been suitably adapted to incorporate the specific channel functions in the forward link, including practical implementation models for the IMUX/OMUX filters and the TWTA. A practical implementation setup based on a memory polynomial model is also discussed. Comprehensive modelling of the Ka-band satellite forward link channel is performed, including the IMUX and OMUX filter magnitude and group delay responses, the TWTA amplitude and phase transfer characteristics, the aggregate phase noise power spectral density mask of the up- and down-conversion stages, and the additive white Gaussian noise (AWGN) at the low-noise amplifier (LNA) of the receiver. The performance of the novel receiver is evaluated for the singlecarrier VSAT reference scenario specified in the DVB-S2X implementation guidelines [3]. A single-carrier TDM waveform with forward error correction (FEC) by means of LDPC codes is employed with a higher baud rate of $34 \mathrm{MBaud}$ and a low carrier roll-off factor of $5 \%$ in a $36-\mathrm{MHz}$ transponder, and it is compared to a lower baud rate of 27.5 MBaud and $20 \%$ roll-off, in order to evaluate the performance in setups with higher and lower linear distortion. The robustness of the detector in the cancellation loop with simple ML demodulation is compared against a receiver structure with soft information exchange with an LDPC decoder [17], showing only marginal degradation of performance. In addition, the convergence of the performance of the proposed receiver is presented for a higher number of iterations of distortion cancellation. In this paper, the packet-error rate (PER) performance of the improved receiver is compared against a number of predistortion techniques at the transmitter, including dynamic data pre-distortion, successive data pre-distortion, and standard static data pre-distortion, showing significant energy efficiency gains. The joint application of successive data pre-distortion and iterative symbol-based equalization demonstrates the best performance in the considered setup, significantly reducing the gap to the AWGN channel. The simulations are performed at practical OBO settings, as well as high OBO settings, in order to evaluate the capability of the techniques to compensate nonlinear distortion. Finally, the complexity of the studied channel compensation techniques is evaluated to provide a reference for satellite model manufacturers. The proposed equalizer is particularly suitable for application in TDM waveforms with low roll-off factors and high symbol rates close to the limits of the transponder bandwidth, enabling the application of higher order modulation and improving the spectral efficiency.

The rest of the paper is organized as follows. Section II describes the satellite system model, including the transmission chain, the channel modelling, and the channel compensation techniques. Section III describes the operation of the proposed equalizer. Section IV presents the performance evaluation results, in particular the PER comparison of ML demodulation with LDPC decoding in the cancellation loop, the PER comparison with data pre-distortion, and the computational complexity. Finally, Section V concludes the paper.

\section{Satellite System Model}

In a satellite communication system, a gateway relays message signals designated to user terminals over a satellite. In this section, the satellite transmission chain in the forward link, the satellite channel model, and a review of the channel compensation techniques are presented.

\section{A. Satellite Transmission Chain}

The block diagram of the satellite transmission chain is presented in Fig. 1. At the gateway transmitter, a stream of data packets with 1504 bits per packet are encoded by means of a FEC scheme. In DVB-S2X [1], first, a Bose-ChaudhuriHocquenghem $(\mathrm{BCH})$ encoder is applied, followed by an LDPC encoder and bit interleaver. The resulting FEC frame with length of up to 64800 bits is mapped to symbols, using APSK modulation with constellations up to 256-APSK at the modulator. In the VSAT scenario, the symbols are grouped in bundled physical-layer (PL) frames with up to 67920 symbols per PL frame, which are in term grouped in superframes with 612540 symbols per superframe. A key feature introduced with the superframe format are the P2 modulated pilot symbols included in each DVB-S2X bundled PL frame [1]. These pilots are inserted after the PL header, and are modulated similarly to the modulation in the corresponding bundled PL frame, 


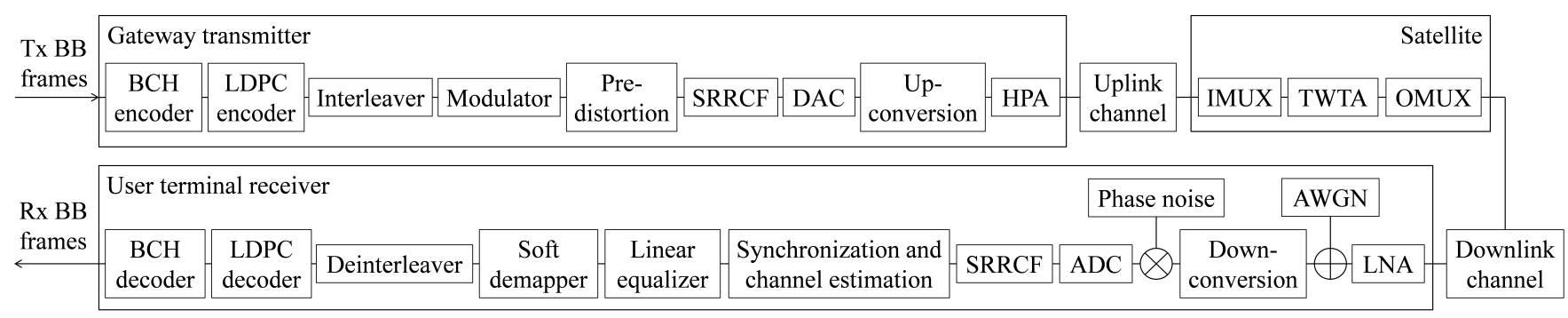

Fig. 1: Block diagram of the satellite transmission chain in the forward link.

representing all constellation points. The information on these pilots can be used in advanced channel estimation techniques, e.g., for estimation of the received constellation centroids used as a reference in the soft demapper and decoder at the receiver, or in the training of channel models used in a variety of channel compensation techniques.

The DVB-S2X transmitter employs static data predistortion [3], [4], [5], [11] to compensate the non-linear distortion effects. The symbols are pulse shaped by means of a square root raised cosine filter (SRRCF), in order to ensure spectrum integrity of the TDM waveform for RF transmission over the feeder link. In the analog front-end, after digital-toanalog conversion (DAC), the baseband signal is up-converted to the carrier frequency, e.g., in the Ka band. Finally, the signal is amplified by means of a high-power amplifier (HPA) at the gateway, and is transmitted over the feeder link through the uplink atmospheric channel.

At the transponder on board the satellite, the signal is passed through an IMUX filter, is amplified by a TWTA, and is passed through an OMUX filter in order to guarantee spectrum integrity for transmission over the user link. Here, the imperfect frequency responses of the IMUX and OMUX filters introduce linear distortion in the form of ISI, while the TWTA introduces non-linear distortion. The signal is then transmitted over the downlink atmospheric channel.

At the user terminal receiver, the signal is amplified by means of an LNA, whereby AWGN is introduced. During down-conversion to baseband, the signal is distorted by phase noise. Next, an analog-to-digital converter (ADC) and a matched SRRCF are applied. After timing and frame synchronization and channel estimation, the symbols are passed through a linear equalizer to counter the linear distortion. A fractionally-spaced linear equalizer with 42 complex-valued taps is applied, working at twice the symbol rate. The symbol vector at the output of the linear equalizer can be expressed as $\mathbf{x}^{(0)}=\operatorname{Eq}\{\mathbf{y}\}=\mathbf{e} * \mathbf{y}$, where $\operatorname{Eq}\{\cdot\}$ is the equalizer operator, $*$ is the linear convolution operator, $\mathbf{y}$ is the symbol vector at the input of the linear equalizer, and $\mathbf{e}$ is the vector with the equalizer taps. These taps are updated in a data-aided manner over the standard P pilot symbols [1], e.g., by using the following adaptive recursive algorithm [12]:

$$
\mathbf{e}_{k+1}=\mathbf{e}_{k}-\mu\left(\mathbf{e}_{k} \mathbf{y}_{k}^{T}-x_{k}\right) \mathbf{y}_{k}^{*},
$$

where $\mathbf{e}_{k+1}$ is the updated equalizer tap vector after the $k$ th pilot $x_{k}, \mathbf{e}_{k}=\left[e_{0}, e_{1}, \ldots, e_{N_{\mathrm{EQ}}-1}\right]$ contains $N_{\mathrm{EQ}}$ taps, initialized as the zero vector $\mathbf{e}_{0}=[0, \ldots, 0]$. The window vector containing $N_{\mathrm{EQ}}$ samples of the received symbol vector $\mathbf{y}$ is denoted as $\mathbf{y}_{k}=\left[y_{k}, y_{k-1}, \ldots, y_{k-N_{\mathrm{EQ}}}\right]$, and is initialized as $\mathbf{y}_{0}=\left[y_{0}, 0, \ldots, 0\right]$. Here, $(\cdot)^{\mathrm{T}}$ is the transpose operator, and $(\cdot)^{*}$ denotes complex conjugation. With values between 0 and 1 , the step size $\mu$ represents a trade-off between convergence speed and stability, e.g., $\mu=0.005$. After linear equalization, the symbols are downsampled to the Nyquist rate. Finally, the demodulator and the decoder recover the received data packets. Here, a soft demapper generates log-likelihood ratios (LLRs), which after deinterleaving are provided as input to the decoding stages, i.e., an LDPC decoder, followed by a $\mathrm{BCH}$ decoder.

A number of simplifications of the satellite transmission chain in the forward link can be considered, preserving all key aspects that affect the physical layer performance. The free-space signal propagation can be characterized as a slowfading process due to rainy weather, and is handled by means of adaptive coding and modulation (ACM), as well as a fading margin in the link budget. The flat gain of the atmospheric satellite channel is also a factor in the link budget, included in the calculation of the available SNR at the receiver. Since the physical layer performance is evaluated as a function of the SNR, unity atmospheric channel gain is considered in the simulations presented in Section IV. Since the HPA at the gateway is operated in the linear regime, the nonlinearity in the satellite channel is introduced primarily by the TWTA. The transmitter and receiver are assumed to be fine synchronized, i.e., genieaided synchronization is performed based on correlation with the transmitted signal, ideally assumed to be known.

As a result, the received PL frame at the input of the demodulator at the user terminal receiver can be expressed as:

$$
\begin{aligned}
\mathbf{x}^{(0)}= & \operatorname{Eq}\left\{\mathbf { h } _ { \mathrm { SRRCF } } * \left[\mathbf { h } _ { \mathrm { OMUX } } * F \left(\mathbf{h}_{\mathrm{IMUX}} *\right.\right.\right. \\
& \left.\left.\left.* \mathbf{h}_{\mathrm{SRRCF}} * \operatorname{Pre}\{\mathbf{x}\}\right)+\mathbf{w}\right] \circ \exp (-j \boldsymbol{\psi})\right\},
\end{aligned}
$$

where $\mathbf{x}$ is the transmitted symbol vector in baseband, Pre $\{\cdot\}$ is the applied pre-distortion operator, $\mathbf{h}_{\mathrm{SRRCF}}$ is the impulse response vector of the SRRCF filter, and $\mathbf{h}_{\text {IMUX }}$ is the impulse response of the IMUX filter. The non-linear transfer function of the TWTA is denoted as $F(\cdot), \mathbf{h}_{\mathrm{OMUX}}$ is the impulse response of the OMUX filter, $\mathrm{w}$ is the AWGN vector, $\psi$ is the phase deviation vector, and $\circ$ denotes element-wise multiplication. In this model, the transmitted signal $\mathbf{x}$ has an average energy per symbol of $E_{\mathrm{s}}$, and the AWGN w has a power spectral density of $N_{0}$. In addition, the responses of 
the SRRCFs and the IMUX/OMUX filters are normalized for unity average power. The same applies for the pre-distorter and the equalizer. Finally, the amplifier function is normalized for unity peak power, and the phase noise is defined by means of a power spectral density mask.

\section{B. Satellite Channel Modelling}

In the satellite forward link, the main sources of distortion are the imperfect characteristics of the IMUX and OMUX filters and the non-linear TWTA response. The signal is also distorted by phase noise due to local oscillator instabilities during the up- and down-conversion processes. In addition, AWGN is introduced at the LNA of the receiver.

1) IMUX and OMUX Filters: Measured magnitude and group-delay responses of the IMUX and OMUX filters in the satellite transponder have been provided as a reference in the DVB-S2X implementation guidelines [3]. While the guidelines only specify the set of characteristics of a $36-\mathrm{MHz}$ transponder, the filter responses are equally applicable to a $225-\mathrm{MHz}$ transponder by using appropriately specified scaling factors for the frequency axis. A computationally efficient and stable implementation of the measured filter responses can be obtained by means of a Chebyshev Type II infinite impulse response (IIR) filter. Synthesized magnitude and group-delay responses of the IMUX and OMUX filters are presented in Fig. 2, using the cheby2 function of the MathWorks MATLAB signal processing toolbox. A 7th-order Chebyshev Type II filter with $-34-\mathrm{dB}$ ripple and $23-\mathrm{MHz}$ edge frequency is used for the IMUX filter, while a 5th-order Chebyshev Type II filter with $-38-\mathrm{dB}$ ripple and $28.6-\mathrm{MHz}$ edge frequency is considered for the OMUX filter. They show to be a good match when compared to the measured responses from [3]. The resulting digital impulse responses, $\mathbf{h}_{\mathrm{IMUX}}$ and $\mathbf{h}_{\mathrm{OMUX}}$, have been generated using $8 \times$ oversampling, and are convolved with a digital representation of the signal. The IMUX/OMUX filters introduce linear distortion in the form of memory effects due to frequency selectivity at high baud rates, which corrupts the matched filtering at the receiver and amplifies the AWGN.

2) TWTA: In this study, a practical model of a non-linear Ka-band linearized TWTA in the satellite forward link is considered. The input amplitude/output amplitude (AM/AM) and input amplitude/output phase (AM/PM) characteristics are presented in Fig. 3, and are denoted as $A(\cdot)$ and $\phi(\cdot)$, respectively. They only depend on the signal amplitude. As a result, the output signal after the TWTA, $F(s)$, can be expressed as $F(s)=A(|s|) \exp [j \phi(|s|)+j \arg (s)]$, where $s$ is the input signal. In this study, $F(s)$ is evaluated using linear interpolation or extrapolation on the measured AM/AM and $\mathrm{AM} / \mathrm{PM}$ characteristics.

In order to facilitate an efficient energy consumption on board the satellite, it is desirable to operate the TWTA close to saturation. In this non-linear regime, signals from higher order APSK constellations are known to suffer from non-linear distortions. Therefore, an OBO is applied to shift the power transfer towards the linear regime at the cost of a penalty in the energy efficiency. The OBO is defined as the ratio
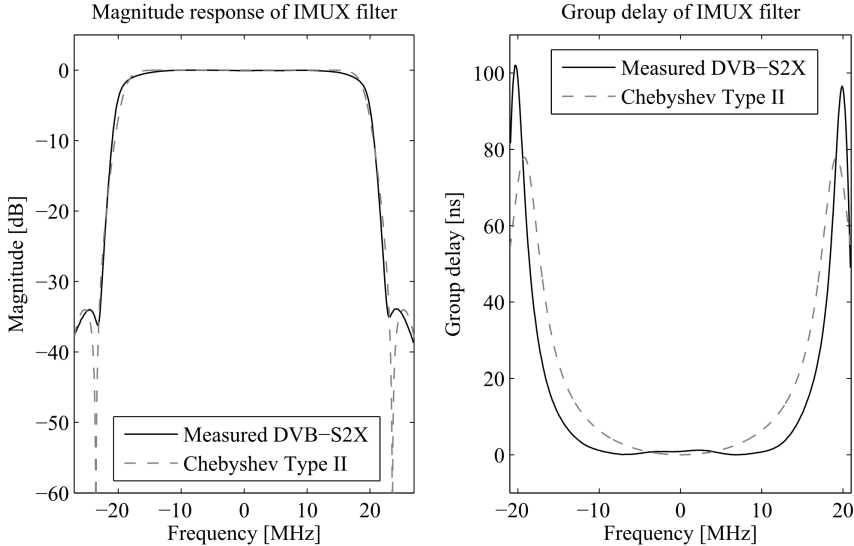

(a) Magnitude and group delay responses of IMUX filter.
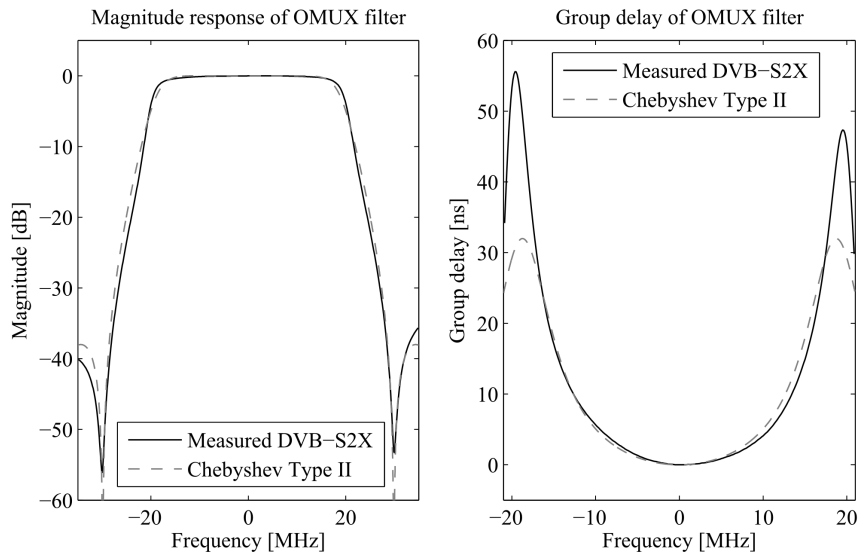

(b) Magnitude and group delay responses of OMUX filter.

Fig. 2: Filter characteristics of a $36-\mathrm{MHz}$ satellite transponder in the forward link.

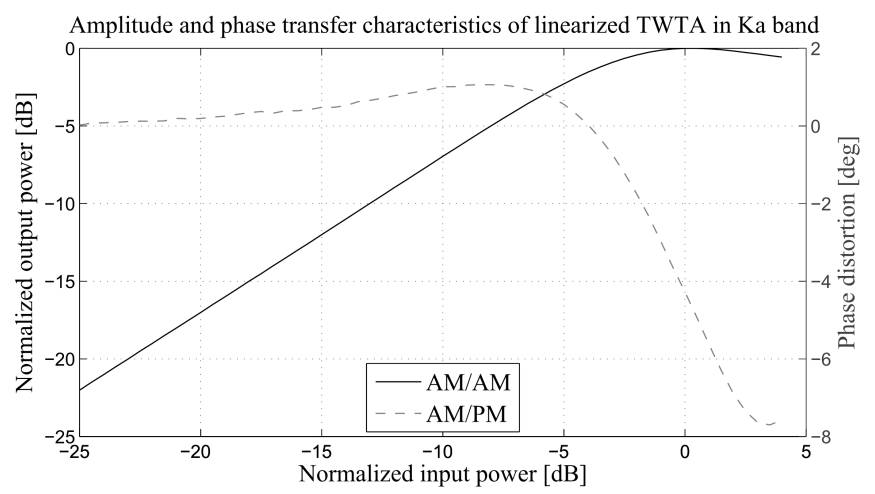

Fig. 3: AM/AM and AM/PM transfer characteristics of a Kaband linearized TWTA.

between the peak output power of the amplifier and the average output power of the signal in the linear domain [14]. The nonlinear distortion introduced by the TWTA can be described as constellation warping and symbol clustering at the input of the demodulator at the receiver [4]. The interfering component is dependent on the signal waveform, and therefore contains useful information. In single-carrier digital transmission schemes, as well as in multi-carrier transmission with a small number of carriers, the interfering component is correlated with the trans- 


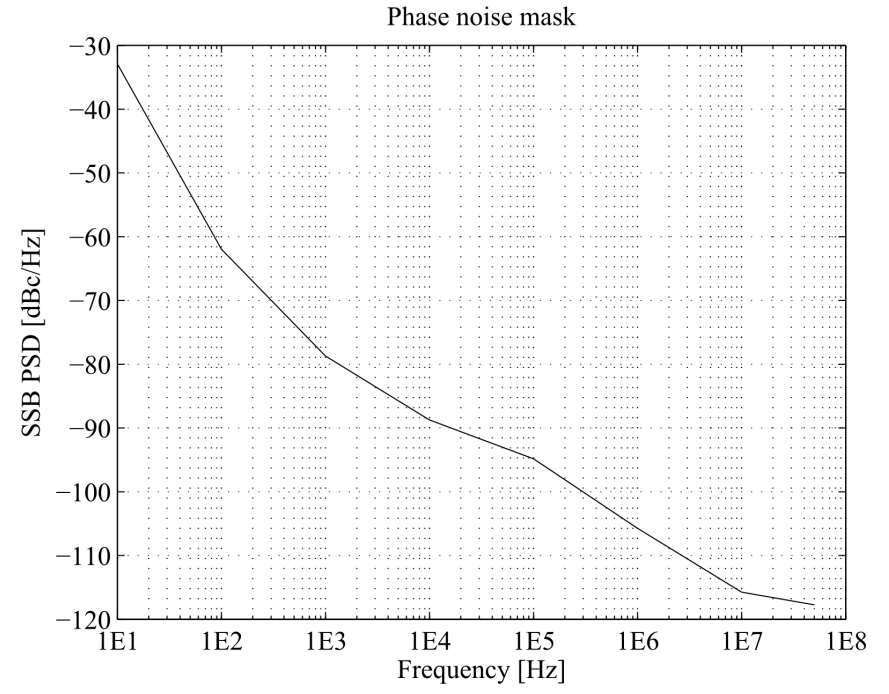

Fig. 4: Phase noise power spectral density mask of the Kaband VSAT scenario in the satellite forward link.

mitted symbols. The received symbols can be decomposed in an attenuated and rotated replica of the transmitted symbols plus non-linear distortion noise. Each received centroid can be individually warped, i.e., it is scaled by a complex-valued factor relative to the transmitted constellation point. There is no general assumption on the distribution of the non-linear distortion noise.

3) $A W G N$ and Phase Noise: At the user terminal receiver, the RF signal is first amplified by means of an LNA. Here, AWGN with double-sided power spectral density $N_{0} / 2$ is introduced to the bandpass information signal. The equivalent baseband signal collects AWGN with one-sided power spectral density $N_{0}$ [12]. After the down-conversion stage, the signal is distorted by phase noise due to local oscillator instabilities. The used aggregate single-sided power spectral density mask of the phase noise is presented in Fig. 4, corresponding to the Ka-band VSAT scenario [3]. According to the procedure outlined in Annex G of [3], the phase deviation process can be modelled as a colored Gaussian process. Under the assumption of small signals, the double-sided power spectral density of this colored Gaussian process corresponds to the single-sided power spectral density mask of the phase noise. The generated phase deviation samples modulate a complex phasor, which is then multiplied with the signal. The impact of phase noise, in addition to the AWGN, stretches the distribution of the noise at the receiver along the constellation ring, degrading the performance of the demodulator.

\section{Channel Compensation Techniques}

The non-linear compensation solutions include a variety of pre-distortion techniques at the transmitter. Signal predistortion [5] is commonly used for amplifier linearization by means of analog electronics that implement the inverse of the amplifier characteristic. Signal pre-distortion can also be applied digitally, i.e., on the samples of a pulse shaped signal immediately before the DAC in the transmitter chain. Nowadays, analog pre-distortion is less common than digital methods. Signal pre-distortion is known to introduce unwanted out-of-band radiation to be suppressed by a transmit filter. A recently proposed solution against spectral regrowth is bandlimited signal pre-distortion [9], [10] which applies digital filters to the out-of-band components and compensates the effect of the non-linear device on the in-band portion of the signal.

Data pre-distortion [4], [5], [6], [7], [8], [11] is a purely digital technique that preserves the signal spectrum, and it is applied to the constellation symbols prior to pulse shaping. Static data pre-distortion [3], [4], [5], [11] accounts for static nonlinearities in the channel, whereby the transmit constellation is modified such that the received centroids are close to the original transmit constellation. The pre-distorted constellation points are stored in a look-up table (LUT). Dynamic data predistortion [4], [11] compensates in addition the memory effects in the channel. It takes into account the current symbol, as well as $(L-1) / 2$ symbols preceding and succeeding each symbol, where $L$ is the memory depth. As a result, the size of the LUT is increased to $M^{L-1}$, where $M$ is the modulation order, for every utilized constellation. Given the already high number of constellations in the DVB-S2X standard, the size of the LUT may require impractically large storage memory.

In general, the more carriers are multiplexed, the lower the constellation warping at the receiver, and the clustering is transformed into Gaussian noise. For a small number of carriers, the signals on the individual carriers become correlated after the non-linear device. As a result, in the case of LUT-based pre-distorters, the size of the LUT increases dramatically, as it needs to store the pre-distorter information for all combinations between the signals, i.e., used constellations.

As an alternative to LUT-based pre-distortion, the dynamic pre-distorter functions can also be computed adaptively using indirect learning [6], [7] or direct learning algorithms [7]. For this purpose, a model for the non-linear channel with memory is required, e.g., a Volterra series [18] or a memory polynomial model [6], [7], [19]. In data pre-distortion algorithms, these models include the linear and non-linear blocks along the chain, e.g., the pulse shaping SRRCF at the transmitter, the IMUX/OMUX filters, the TWTA, and the matched SRRCF at the receiver. In order to train the model coefficients, a dedicated training sequence is transmitted and fed back from the receiver. This can be practically implemented by means of the P2 pilot fields in the superframe format. As another alternative solution, successive data pre-distortion has been introduced in [8] for multi-carrier transmission. This technique employs the measured IMUX/OMUX and TWTA characteristics, as well as the impulse responses of the SRRCFs at the transmitter and the receiver, to iteratively minimize the interfering component.

The symbol-based equalizer with non-linear distortion cancellation proposed in this study as an addition to the user terminal receiver in the satellite forward link is a form of decisiondirected equalizer [12]. This equalizer has been shown to be an effective technique to compensate the interfering component in a non-linear channel, and to significantly reduce the SNR penalty with respect to the linear channel [14], [15], [17], [20]. It is the objective of this paper to compare the performance of 
the proposed equalizer with state-of-the-art dynamic data predistortion, successive data pre-distortion, as well as static data pre-distortion, and to show that the best system performance is achieved when both pre-distortion at transmitter and decisiondirected equalization at the receiver are applied. In addition, the performance of a simple ML demodulator in the detector in the cancellation loop is compared against an LDPC decoder, in order to show the robustness of the proposed equalizer to decision errors.

\section{ITERATIVE EQUALIZATION AND CANCELLATION OF NON-LINEAR DISTORTION}

In this section, the background and operation of the symbolbased equalizer with non-linear distortion cancellation are discussed. The building blocks in the forward link setup, including two equalizer architectures are defined. These include an ML demodulator with hard decision $(\mathrm{Rx} 1)$ and a soft information exchange with the LDPC decoder ( $\mathrm{Rx} 2)$ in the cancellation loop.

\section{A. Background and Motivation}

Decision-directed detection by means of turbo equalization has been proposed for application in non-linear satellite channels in [13]. A channel model based on Volterra series is assumed, and minimum mean-squared error (MMSE) filters are computed to cancel the non-linear terms in an iterative fashion, using soft information exchange with the LDPC decoder. In [14], a novel symbol-based iterative equalization scheme has been introduced which reduces the complexity of the receiver by means of a simplified model for the received symbols after the non-linear device, and by using ML demodulation with hard decision in the cancellation loop. Instead of computing MMSE filters for every component in the Volterra series, a simple procedure can be applied to reconstruct and cancel the non-linear noise component. At the heart of the simplification is a first-order decomposition of the received symbols at the input of the demodulator, based on a symbol scaling factor representing the warping effect and a non-linear noise component containing the higher nonlinear orders. In addition to non-linear compensation, memory effects in the form of ISI are also taken into account in the reconstruction and cancellation of the interfering component due to the fact that the equalizer operates on a large block of symbols, e.g., a PL frame.

The proposed equalizer estimates and cancels the interference in the non-linear channel with memory based on detection of the symbols at the receiver. The receiver in the return satellite link, e.g., a gateway station, receives all the signals, and therefore it can compensate adjacent channel interference $(\mathrm{ACI})$ in the form of intermodulation interference (IMI) between the carriers in a multi-carrier scenario. In the forward satellite link, however, a receiver, e.g., a user terminal, normally receives the signal on a single carrier, unless cooperation between receivers is assumed. As a result, it can only compensate the self-interference within that carrier, in the form of ISI, caused during the transmission over the non-linear channel with memory. Therefore, the proposed

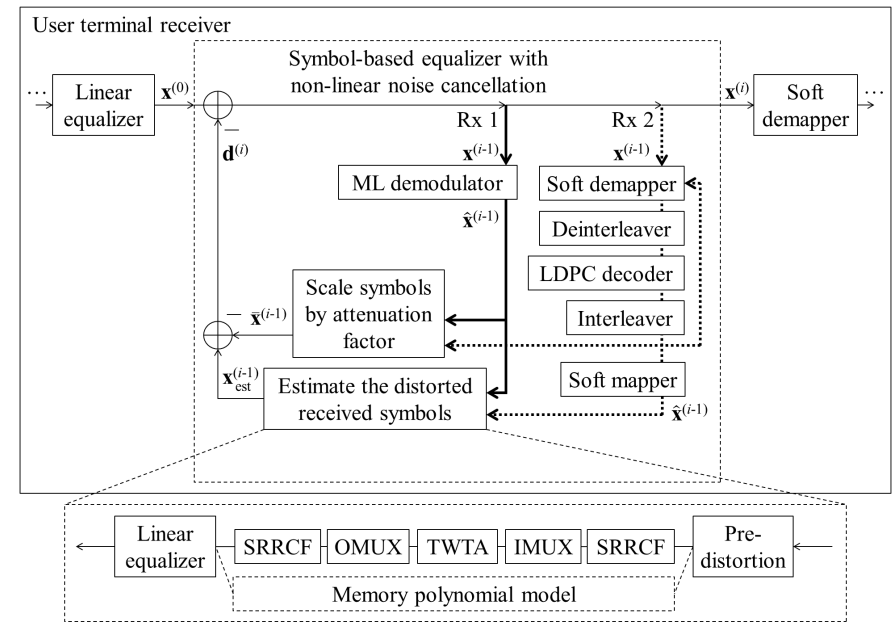

Fig. 5: Block diagram of the symbol-based equalizer with nonlinear distortion cancellation in the satellite forward link, using an ML demodulator (Rx 1 - bold solid lines) or an LDPC decoder ( $\mathrm{Rx} 2$ - bold dotted lines) in the cancellation loop. The estimate of the distorted received symbols can be obtained by either using the characteristics of the IMUX/OMUX filters and TWTA or a memory polynomial model.

equalizer is best suitable for single-carrier scenarios in the satellite forward link. It can also be applied in a multi-carrier scenario in combination with a pre-distortion technique at the transmitter that compensates the IMI. In this case, the proposed equalizer can further minimize the residual self-interference within the received carrier.

\section{B. Function}

The block diagram of the symbol-based equalizer with non-linear distortion cancellation is presented in Fig. 5. The symbols at the output of the linear equalizer, downsampled to the Nyquist rate, are provided as input to the iterative nonlinear equalizer. At this point, a set of received symbols, e.g., a received PL frame $\mathbf{x}^{(0)}$, is buffered. As a first step of the processing, detection is performed with the received symbols, whereby two receiver structures are evaluated in this study. The performance of a simple ML demodulator in the detector in the cancellation loop is compared against an LDPC decoder, in order to show the robustness of the proposed equalizer to decision errors.

In the first receiver $(\mathrm{Rx} 1)$, the symbols are ML demodulated by means of a hard decision using the received constellation centroids as a reference. Here, the P2 modulated pilot symbols in each DVB-S2X bundled PL frame [1], as part of the superframe format, are utilized in order to estimate the received constellation centroids. The vector of detected symbols used in the $i$ th iteration is denoted as $\widehat{\mathbf{x}}^{(i-1)}=\operatorname{Demod}\left[\mathbf{x}^{(i-1)}\right]$.

In the second receiver ( $\mathrm{Rx} 2$ ), the LDPC decoder is included in the cancellation loop in accordance with the setup of the turbo equalizer [13]. The detected symbols are therefore obtained after soft information exchange with the decoder, i.e., after soft demapping, deinterleaving, LDPC decoding, interleaving and soft mapping are applied to the symbols 
$\mathbf{x}^{(i-1)}$. Here, the soft demapper provides extrinsic LLRs to the decoder via deinterleaver, using the received constellation centroids as reference, and employs the interleaved LLRs from the previous decoding iteration as a priori information [13]. The interleaved decoder LLRs are also used in the soft mapper to compute bit probabilities $\operatorname{Pr}\left\{a_{n, q}=b_{m, q}\right\}$, where $a_{n, q}$ are the bits corresponding to a symbol $x_{n}^{(i-1)}, b_{m, q}$ are interleaved source bits corresponding to a constellation point, $c_{m}, q=1, \ldots, \log _{2}(M), m=1, \ldots, M$, where $M$ is the modulation order. Under the assumption of bit independence, these probabilities are used to compute soft detected symbols as follows:

$$
\widehat{x}_{n}^{(i-1)}=\sum_{m=1}^{M} c_{m} \prod_{q=1}^{\log _{2}(M)} \operatorname{Pr}\left\{a_{n, q}=b_{m, q}\right\} .
$$

The estimate of the interfering component is obtained through subtraction of two terms. To obtain the first term, in $\mathrm{Rx} 1$, the detected symbols are scaled by individual attenuation factors, $K_{c_{m}}$, according to the respective constellation point $c_{m}$, to obtain $\overline{\mathbf{x}}^{(i-1)}$, where $\bar{x}_{c_{m}, n}^{(i-1)}=K_{c_{m}} \widehat{x}_{c_{m}, n}^{(i-1)}$. This is essential to account for the constellation warping effects. The individual scaling factors represent the ratio of a received centroid to the corresponding transmitted constellation point. In $\mathrm{Rx} 2$, soft scaled symbols are calculated as follows:

$$
\bar{x}_{n}^{(i-1)}=\sum_{m=1}^{M} K_{c_{m}} c_{m} \prod_{q=1}^{\log _{2}(M)} \operatorname{Pr}\left\{a_{n, q}=b_{m, q}\right\} .
$$

To obtain the second term, the symbols $\widehat{\mathbf{x}}^{(i-1)}$ are used to estimate the distorted received symbols based on models of the distortion functions and the processing blocks along the transmission chain. Here, only models for the distortion sources are used, which can be considered deterministic. These include the linear and non-linear distortions in the satellite transponder due to the IMUX filter, TWTA, and OMUX filter. Measured models for the linear and non-linear distortion functions, such as the measured AM/AM and AM/PM characteristics of the TWTA and the measured magnitude and group delay responses of the IMUX and OMUX filters, can be all obtained before the commission of each satellite. In stateof-the-art non-linear compensation techniques, the Volterra series and memory polynomial models [6], [7], [19] can be used to describe the non-linear channel with memory. These models can be obtained at the receiver by means of training data, and they can follow the slow time-variant changes in the transponder characteristics, such as diurnal changes in the responses of the analog filters or variations in the OBO. In practice, a sufficiently accurate channel model can be obtained, using the P2 pilot fields in the DVB-S2X superframe format, by means of the memory polynomial model with higher nonlinear order and higher memory depth. Therefore, under the assumption of accurate channel estimation, the amplitude and phase characteristics of the TWTA and IMUX/OMUX filters from Section II-B are directly used in the proposed equalizer in this study, as an equivalent to the memory polynomial model, to obtain an estimate of the the received symbols.

In addition, if the application of a pre-distorter at the trans- mitter is desirable in conjunction with the non-linear equalizer at the receiver, the setup of the pre-distortion operation is made known to the receiver, e.g., as information in the frame transmission preamble, frame header, or dedicated signalling channel. For completeness, the pre-distortion block is included in the setup of the non-linear equalizer in Fig. 5.

Therefore, in order to estimate the distorted received symbols, the symbols $\widehat{\mathbf{x}}^{(i-1)}$ are first passed through the known pre-distorter block. The symbols are then pulse shaped, and the models for the IMUX filter, TWTA (maintaining the operating point), and OMUX filter are applied. As part of the receiver chain, the symbols are match-filtered, and the known linear equalizer block is applied. The estimate of the received symbols can be expressed as:

$$
\begin{aligned}
\mathbf{x}_{\mathrm{est}}^{(i-1)}= & \operatorname{Eq}\left\{\mathbf{h}_{\mathrm{SRRCF}} * \mathbf{h}_{\mathrm{OMUX}} *\right. \\
& \left.* F\left(\mathbf{h}_{\mathrm{IMUX}} * \mathbf{h}_{\mathrm{SRRCF}} * \operatorname{Pre}\left\{\widehat{\mathbf{x}}^{(i-1)}\right\}\right)\right\} .
\end{aligned}
$$

The scaled symbols are subtracted from the estimate of the received symbols to reconstruct an estimate of the non-linear distortion noise as:

$$
\mathbf{d}^{(i)}=\mathbf{x}_{\mathrm{est}}^{(i-1)}-\overline{\mathbf{x}}^{(i-1)} .
$$

This estimate of the interfering component is subtracted from the buffered symbols to obtain a new set of the received symbols as output of the first iteration of non-linear distortion cancellation. In the second iteration, the newly obtained received symbols are detected and used to better estimate the interfering component, which is again subtracted from the originally buffered received symbols. The improved vector of received symbols after the $i$ th iteration can be expressed as:

$$
\mathbf{x}^{(i)}=\mathbf{x}^{(0)}-\mathbf{d}^{(i)}
$$

The output of this process are the newly obtained received symbols after a number of iterations. It is shown in Section IV that even one iteration can yield a significant energy efficiency gain. Finally, the buffer is released and a new frame of received symbols is processed. As the iterative symbol-based equalizer outputs symbols with reduced interference, more reliable LLRs can be computed at the subsequently applied soft demapper, improving the performance of the decoder.

\section{Performance Evaluation}

In this section, PER performance of the iterative symbolbased equalizer applied at the receiver is studied for application in the single-carrier VSAT forward satellite link. First, the robustness of the detector in the cancellation loop to decision errors is evaluated by comparing the performance of simple ML demodulation with hard decision ( $\mathrm{Rx} 1)$ against soft information exchange with the LDPC decoder ( $\mathrm{Rx} 2)$. The convergence of the iterative distortion cancellation approach is presented for a higher number of iterations. The PER performance of the proposed equalizer is then compared with a number of non-linear channel compensation techniques at the transmitter, such as dynamic data pre-distortion with memory polynomial model [6], successive data pre-distortion using 
exact transponder characteristics [8], and the reference DVBS2X static data pre-distortion [3], [4], [5], [11]. The joint application of non-linear compensation techniques at both the transmitter and the receiver is also considered, in order to show the highest achievable gains. In all scenarios, a linear equalizer is applied as a standard feature of the DVB-S2X receiver. The proposed iterative symbol-based equalizer is applied immediately after the linear equalizer. Finally, the computational complexity of the proposed equalizer is evaluated and compared to the pre-distortion solutions.

A single-carrier VSAT scenario is considered for the performance evaluation as representative of state-of-the-art interactive satellite networks, specified as one of the four reference scenarios in the DVB-S2X implementation guidelines [3]. The satellite channel model consists of the IMUX and OMUX filter characteristics, the transfer characteristic of the linearized TWTA, the phase noise and AWGN. Constellations of 16APSK and 32-APSK are simulated, whereby one modulation order is applied per PL frame. A carrier symbol rate of 27.5 MBaud is applied with $20 \%$ roll-off factor as a legacy scenario to DVB-S2 [21], while also $34 \mathrm{MBaud}$ with $5 \%$ roll-off factor is considered as an example of high carrier symbol rate with low roll-off factor in the transponder bandwidth of $36 \mathrm{MHz}$. In this way, the performance of the channel compensation techniques can be studied in a scenario with lower linear distortion and a scenario with higher linear distortion. In addition, nonlinear distortion is added by operating the TWTA at low OBO settings. Due to unavailability of directly comparable results from the DVB-S2X implementation guidelines for the singlecarrier VSAT scenario, the optimum OBO values of $1.6 \mathrm{~dB}$ and $2.2 \mathrm{~dB}$ for 16 -APSK and 32-APSK, respectively, are borrowed from the single-carrier DTH scenario [3]. A higher OBO setting of $10 \mathrm{~dB}$ is also considered in the comparison with successive data pre-distortion, in order to provide a reference scenario with only linear distortion, and thus facilitate the evaluation of the capability of the pre-distortion and equalization techniques to compensate non-linear distortion in the presence of memory effects. It should be noted, that while the the DVB-S2X implementation guidelines [3] suggest a direct up-scaling of the symbol rates and filter characteristics from a $36-\mathrm{MHz}$ transponder to a $225-\mathrm{MHz}$ transponder, the implementation of a linearized wide-band amplifier may have a frequency response different from the assumed memoryless model, resulting in further distortion and degradation of the performance of the receiver.

There are a number of processing blocks used in the compared algorithms which require training procedures summarized as follows:

- In the scenario with static data pre-distortion and linear equalization, these include the taps of the linear equalizer and the pre-distorted constellation for static data predistortion. The taps of the linear equalizer can be computed at the receiver by using $\mathrm{P}$ pilots from a quadrature phase-shift keying (QPSK) constellation at the input of the linear equalizer. The pre-distorted constellation is then computed at the transmitter by using fed-back P2 pilots at the output of the linear equalizer at the receiver.

- In the scenario with dynamic data pre-distortion and linear equalization, these are the inverse channel model based on the memory polynomial model and the taps of the linear equalizer. For the training of the inverse channel model, first, the P2 pilots at the input of the linear equalizer at the receiver are fed back to the transmitter, in order to compute the memory polynomial coefficients of the pre-distorter. The taps of the linear equalizer are then computed by means of pre-distorted $\mathrm{P}$ pilots from QPSK constellation at the input of the linear equalizer.

- In the scenarios where the the symbol-based iterative equalizer is applied after the linear equalizer, the received constellation centroids are trained last, and these are obtained from equalized P2 pilot symbols at the output of the linear equalizer. When pre-distortion techniques are applied in conjunction with the symbol-based iterative equalizer, these P2 pilots are also pre-distorted at the transmitter.

\section{A. ML demodulation vs. LDPC decoding in the detector in the cancellation loop}

The detector in the cancellation loop of the proposed symbol-based non-linear equalizer plays the significant role of providing reliable received symbols for estimation and cancellation of the interfering component. A turbo equalizer performs the iterations by exchanging information with the decoder stage. Therefore, it is of interest to compare the performance of the simple ML demodulator ( $\mathrm{Rx} 1)$ against a setup, where the LDPC decoder $(\operatorname{Rx} 2)$ is included in the cancellation loop.

The performance of the channel compensation techniques is evaluated by means of a Monte Carlo simulation of the PER in the satellite forward link. The PER is evaluated as a function of the SNR defined as $P_{\text {sat }} / N$, i.e., the ratio of the on-board TWTA saturation power to the noise power, which can be related to the $E_{\mathrm{s}} / N_{0}$ ratio in the linear domain as follows [3]:

$$
\frac{P_{\mathrm{sat}}}{N}=\frac{E_{\mathrm{s}}}{N_{0}} \frac{R_{\mathrm{s}}}{W_{\text {ref }}} \text { OBO, }
$$

where $R_{\mathrm{S}}$ is the symbol rate and $W_{\text {ref }}$ is the transponder bandwidth in single-carrier mode. A high number of long PL frames are simulated, e.g., up to $10^{6}$ packets per simulation point, resulting in sufficient convergence of the statistics down to a PER of $10^{-3}$.

The results for 1 and 2 iterations of non-linear distortion noise cancellation are presented in Fig. 6. There is a minor difference between ML demodulation and LDPC decoding within $0.2 \mathrm{~dB}$ across the scenarios. This shows that simple ML demodulation of the received symbols is sufficient in the context of the proposed equalizer in this scenario, resulting in significant reduction of the computational complexity of the receiver. In addition, a higher number of iterations of $\operatorname{Rx} 1$, i.e., 4, 5 and 10 iterations, have also been simulated in the scenario with 32-APSK, 34 Mbaud symbol rate and 5\% rolloff. The results confirm the stable and rapid convergence of the performance for higher number of iterations with negligible improvements beyond 2 iterations. In the following, only $\mathrm{Rx}$ 1 is considered in the simulations of the iterative symbol-based equalizer. 


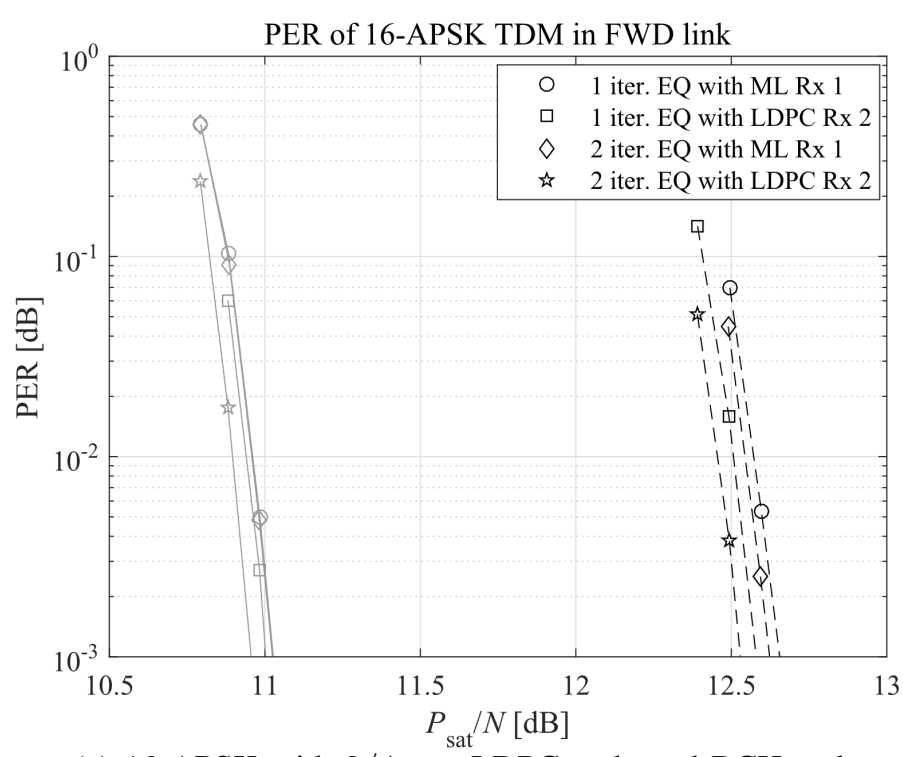

(a) 16-APSK with 3/4-rate LDPC code and BCH code.

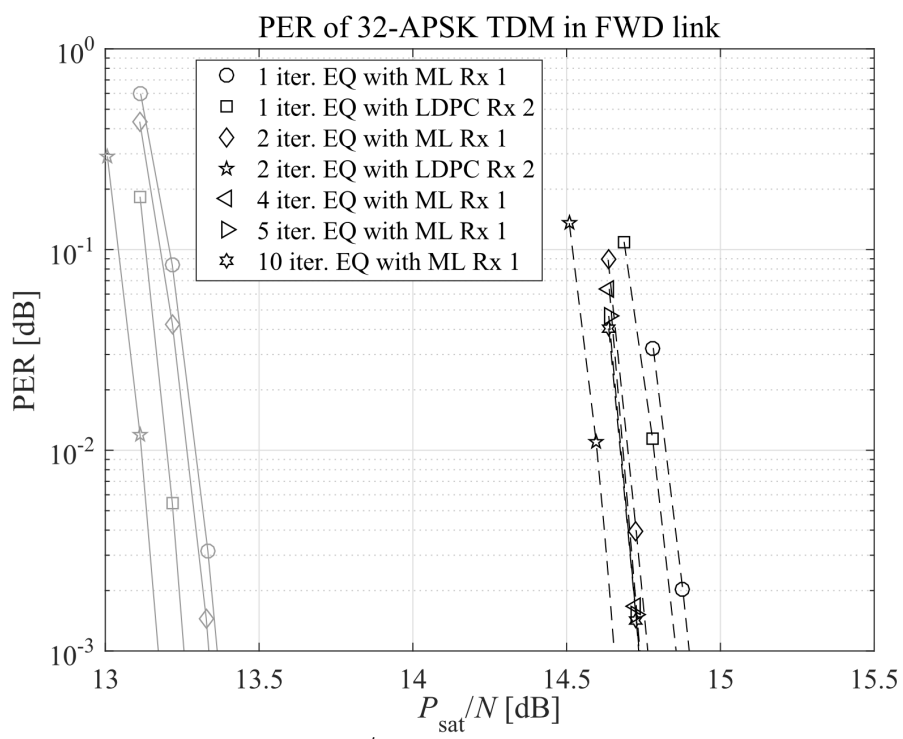

(b) 32-APSK with 3/4-rate LDPC code and BCH code.

Fig. 6: PER of single-carrier TDM with non-linear distortion cancellation at receiver in the satellite forward link at practical OBO, using ML demodulation (Rx 1) or LDPC decoding (Rx 2 ) in the cancellation loop. Solid lines represent the scenario with $20 \%$ roll-off and 27.5 Mbaud symbol rate, while the dashed lines correspond to the scenario with $5 \%$ roll-off and 34 Mbaud symbol rate.

\section{B. Symbol-based equalization and non-linear distortion can- cellation vs. dynamic data pre-distortion}

For compensation of the non-linear channel with memory, dynamic data pre-distortion with indirect learning [6] is applied with memory of $Q=5$ taps and highest non-linear order of $P=5$ in the memory polynomial model, sufficient to well suppress the signal distortions according to [6]. Here, the inversion of the polynomial model is obtained by means of a least squares (LS) approach. The performance of the symbolbased equalizer is evaluated for 1 and 2 iterations of non- linear distortion cancellation. In addition, the performance of the combination of the two solutions is also simulated.

The PER performances of dynamic data pre-distortion (dyn. PD) and symbol-based equalizer with non-linear distortion cancellation (iter. EQ) are compared with conventional static data pre-distortion (stat. PD) in Fig. 7. Dynamic data predistortion provides an SNR gain as compared to static data predistortion. However, even one iteration of non-linear distortion cancellation is sufficient to outperform pre-distortion, and to obtain the majority of the gain. The SNR gains at PER of $10^{-3}$ relative to static data pre-distortion are summarized in TABLE I. These energy efficiency gains are shown to increase for higher-order modulations due to their higher sensitivity to non-linear distortion. In addition, higher gains of the channel compensation schemes can be seen in the scenario with 34-Mbaud symbol rate and 5\% roll-off as compared to the scenario with 27.5 -Mbaud symbol rate and $20 \%$ rolloff, because of the higher linear distortion, due to the higher symbol rate close to the full transponder bandwidth, and the higher non-linear distortion, due to the increased PAPR of the waveform at the lower roll-off factor.

In order to facilitate a fair comparison between scenarios with different non-linear channel characteristics and the performance in the AWGN channel, the residual gain of the TWTA at the considered OBO, $G_{\mathrm{OBO}}$, is defined. This gain represents the ratio between the input back-off (IBO) and the OBO in the linear domain, where the IBO is defined as the ratio between the peak input power of the amplifier and the average input power of the signal. Since practical amplifier characteristics are generally normalized for unity peak input/output power, the following relationship holds: $\mathrm{IBO} \geq \mathrm{OBO}$ for $\mathrm{OBO} \geq 0$ $\mathrm{dB}$. Therefore, the amplifier introduces a residual gain in the $E_{\mathrm{s}} / N_{0}$ and $P_{\mathrm{sat}} / N$ for $\mathrm{OBO}>0$. For a fair comparison, a normalized $E_{\mathrm{s}} / N_{0}$ is defined and used as a comparative metric. It is derived from the $P_{\text {sat }} / N$ requirement, whereby the the influence of the residual gain $G_{\mathrm{OBO}}$ is undone. In general, as the gain of the TWTA reduces the $P_{\text {sat }} / N$ requirement, to undo its influence means to increase the $P_{\text {sat }} / N$ in (8) by $G_{\text {Ово }}$. As a result, the normalized $E_{\mathrm{s}} / N_{0}$ can be defined in the linear domain as follows:

$$
\text { norm. } \frac{E_{\mathrm{s}}}{N_{0}}=\frac{P_{\mathrm{sat}}}{N} G_{\mathrm{OBO}}\left(\frac{R_{\mathrm{s}}}{W_{\text {ref }}} \mathrm{OBO}\right)^{-1} .
$$

The results in this paper are first compared to the $E_{\mathrm{s}} / N_{0}$ requirements of 16-APSK and 32-APSK with a 3/4-rate LDPC code and a BCH code in the AWGN channel [21], i.e., $10.21 \mathrm{~dB}$ and $12.73 \mathrm{~dB}$, respectively. While $P_{\text {sat }} / N=$ $E_{\mathrm{s}} / N_{0}=$ norm. $E_{\mathrm{s}} / N_{0}$ for the AWGN curves in Fig. 7, these SNRs are related via (8) and (9) in the other scenarios. The $P_{\text {sat }} / N$ requirements of the best achievable performance of the channel compensation techniques in the scenario with 27.5 Mbaud symbol rate and 20\% roll-off from Fig. 7 are 10.95 $\mathrm{dB}$ and $13.32 \mathrm{~dB}$ for 16-APSK and 32-APSK, respectively. Using the 1-dB and 1.4-dB gains of the TWTA from Fig. 3 at $1.6 \mathrm{~dB}$ and $2.2 \mathrm{~dB}$ OBO, respectively, the normalized $E_{\mathrm{s}} / N_{0}$ requirements can be computed as $11.52 \mathrm{~dB}$ and $13.69 \mathrm{~dB}$. It is shown that the studied non-linear compensation techniques significantly reduce the gap to the performance in the AWGN 


\begin{tabular}{|l|l|l|l|l|l|l|}
\hline $\begin{array}{l}\text { Scenario with 3/4-rate LDPC code } \\
\text { and BCH code }\end{array}$ & $\begin{array}{l}\text { Required } \\
P_{\text {sat }} / N \text { of } \\
\text { stat. PD [3] }\end{array}$ & $\begin{array}{l}\text { Gain } \\
\text { of dyn. } \\
\text { PD [6] }\end{array}$ & $\begin{array}{l}\text { Gain of } \\
20 \text { iter. } \\
\text { PD [8] }\end{array}$ & $\begin{array}{l}\text { Gain of } \\
2 \text { iter. } \\
\text { EQ Rx 1 }\end{array}$ & $\begin{array}{l}\text { Gain of dyn. } \\
\text { PD [6] + 2 } \\
\text { iter. EQ Rx 1 }\end{array}$ & $\begin{array}{l}\text { Gain of 20 iter. } \\
\text { PD [8] + 2 iter. } \\
\text { EQ Rx 1 }\end{array}$ \\
\hline 16-APSK, 27.5 MBaud, 20\% roll-off & 12.23 & 0.17 & 0.31 & 1.21 & 1.28 & 1.38 \\
\hline 16-APSK, 34 MBaud, 5\% roll-off & 14.8 & 0.69 & 1.37 & 2.19 & 2.28 & 2.5 \\
\hline 32-APSK, 27.5 MBaud, 20\% roll-off & 15.53 & 0.29 & 0.37 & 2.19 & 2.21 & 2.25 \\
\hline 32-APSK, 34 MBaud, 5\% roll-off & 19.54 & 1.71 & 2.8 & 4.8 & 4.81 & 4.95 \\
\hline
\end{tabular}

TABLE I: Required $P_{\mathrm{sat}} / N$ [dB] at $10^{-3}$ PER of static data pre-distortion [3] and relative gains [dB] of dynamic [6]/successive [8] data pre-distortion at the transmitter and non-linear distortion cancellation at the receiver ( $\mathrm{Rx} 1)$ in the single-carrier VSAT satellite forward link at practical OBO.

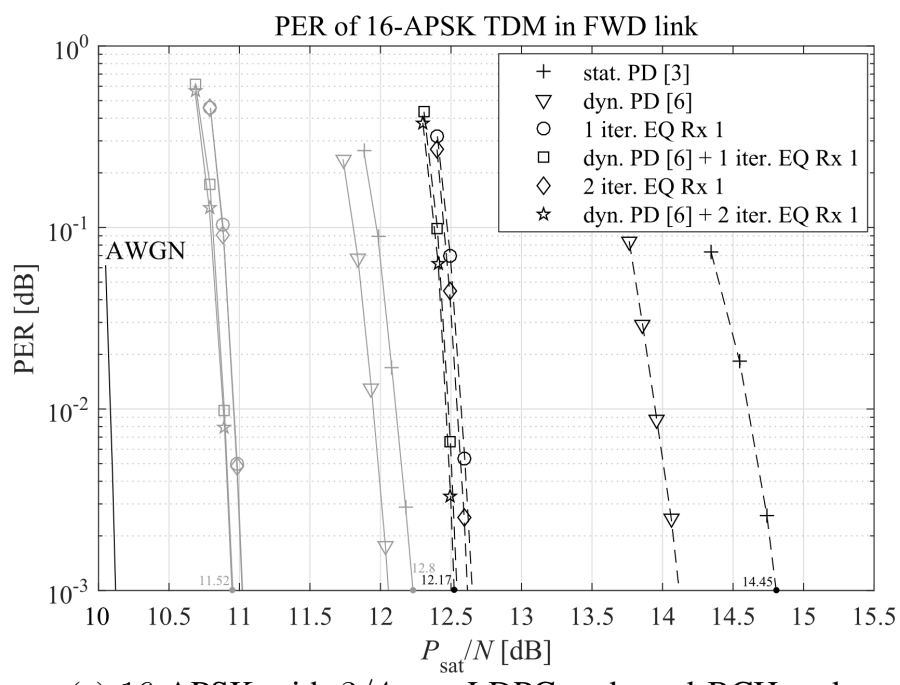

(a) 16-APSK with 3/4-rate LDPC code and BCH code.

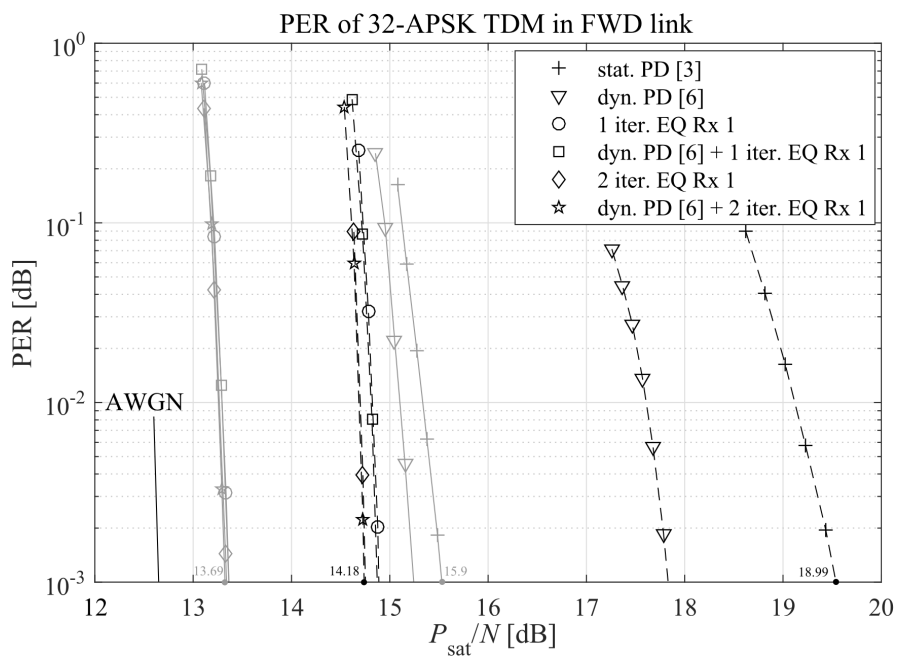

(b) 32-APSK with 3/4-rate LDPC code and BCH code.

Fig. 7: PER of single-carrier TDM with static/dynamic data pre-distortion at the transmitter and/or non-linear distortion cancellation at the receiver in the satellite forward link at practical OBO. Solid lines represent the scenario with $20 \%$ roll-off and 27.5 Mbaud symbol rate, while the dashed lines correspond to the scenario with 5\% roll-off and 34 Mbaud symbol rate. Normalized $E_{\mathrm{s}} / N_{0}$ is presented at selected points. channel from [21] from $2.59 \mathrm{~dB}$ to $1.31 \mathrm{~dB}$ and from 3.17 $\mathrm{dB}$ to $0.96 \mathrm{~dB}$ for 16 -APSK and 32-APSK, respectively. If one executes the same computation for the scenario with 34 Mbaud symbol rate and 5\% roll off, the gap to the AWGN channel is reduced from $4.24 \mathrm{~dB}$ to $1.96 \mathrm{~dB}$ and from 6.26 $\mathrm{dB}$ to $1.45 \mathrm{~dB}$.

While there is no direct comparison for the single-carrier VSAT scenario with the scenarios simulated in [3], the presented results of static data pre-distortion with linear equalization in this paper can be compared with the results reported in [3] in the single-carrier DTH scenario with 34-Mbaud symbol rate and 5\% roll-off for $3 / 4$-rate 16 -APSK. The differences in the satellite link setup between the two scenarios are the conventional (not linearized) TWTA characteristics and the higher phase noise in the DTH scenario. The $P_{\text {sat }} / N$ in the DTH scenario is reported as $13 \mathrm{~dB}$ for an equivalent PER of $10^{-3}$. The compared performance in Fig. 7 for the VSAT scenario is $14.8 \mathrm{~dB}$. In order to compare the two values in terms of normalized $E_{\mathrm{s}} / N_{0}$, the residual gain of the TWTA at the considered OBO needs to be taken into account. In the DTH scenario, the not linearized TWTA has a gain of approximately $4.5 \mathrm{~dB}$ at $1.6 \mathrm{~dB} \mathrm{OBO}$, while the linearized TWTA has a gain of only $1 \mathrm{~dB}$. As a result, it can be argued that the result presented in this paper for the VSAT scenario are $1.7 \mathrm{~dB}$ better in terms of normalized $E_{\mathrm{s}} / N_{0}$ than the result of the DTH scenario in [3]. This difference can be attributed to the lower non-linear distortion due to the use of a linearized TWTA model and lower phase noise in the VSAT scenario.

In order to provide a justification for the numerical accuracy of the results, a number of implementation issues are discussed. First, the implementation of the fractionally-spaced linear equalizer is subject to step size optimization used for the training of the equalizer taps in an adaptive fashion. In addition, the practical implementation of the filter responses by means of Chebyshev Type II approximation offers a slightly higher group delay as compared to the measured responses for the considered symbol rates. Finally, the OBO setting itself can be a source of mismatch, because the DVB-S2X implementation guidelines [3] specify a range of the OBO setting within $0.2-0.3 \mathrm{~dB}$ for the single-carrier DTH scenario. These values have been adopted in this paper for application in the singlecarrier VSAT scenario, due to unavailability of specified values for this case in [3]. As a result, these impairments need to be taken into account when comparing the results to another implementation. All the relevant implementation parameters 
in this paper have been mentioned in the setup definitions in Section II.

\section{Symbol-based equalization and non-linear distortion can- cellation vs. successive data pre-distortion}

The iterative symbol-based equalizer is also compared to successive data pre-distortion [8] applied in the single-carrier VSAT scenario. Instead of using a memory polynomial model to compute the pre-distorter function as in dynamic data predistortion, successive data pre-distortion directly employs the measured characteristics of the TWTA, IMUX, and OMUX filters, as well as the SRRCF responses at transmitter and receiver, in order to estimate and cancel the interfering component. The symbol-based equalizer is applied with 1 and 2 iterations of non-linear distortion cancellation. The iterative data pre-distortion technique is used with 20 iterations of data pre-distortion, following the setup used in [8]. Similar modulation and coding, as well as OBO settings, are considered as in the previous section.

The PER performances of successive data pre-distortion (iter. PD) and symbol-based equalizer with non-linear distortion cancellation (iter. EQ) are compared with conventional static data pre-distortion (stat. PD) in Fig. 8. Iterative successive data pre-distortion shows significant gains with respect to static data pre-distortion, and even outperforms dynamic data pre-distortion. However, iterative symbol-based equalization still proves to be the superior solution, achieving gains with respect to successive data pre-distortion of $0.9 \mathrm{~dB}$ and 1.82 $\mathrm{dB}$ for 16 -APSK and 32-APSK, respectively, in the scenario with 27.5 Mbaud symbol rate and $20 \%$ roll-off, and $0.82 \mathrm{~dB}$ and $2 \mathrm{~dB}$ in the scenario with 34 Mbaud symbol rate and $5 \%$ roll-off. The SNR gains at PER of $10^{-3}$ of the scenarios with successive data pre-distortion are also summarized in TABLE I. The combination of successive data pre-distortion at the transmitter and symbol-based equalization with non-linear distortion cancellation at the receiver presents small additional gains of up to $0.31 \mathrm{~dB}$ across the scenarios as compared to iterative equalization alone, proving the effectiveness of the proposed equalizer. Nonetheless, the best performance in the studied VSAT scenario is achieved with this combination, showing gains of up to $4.95 \mathrm{~dB}$ as compared to standard static data pre-distortion.

Finally, in order to compare the capability of successive data pre-distortion and iterative equalization to compensate the non-linear distortions in the channel, the performance is evaluated in a scenario with only linear distortions by means of simulations at large $\mathrm{OBO}$ of $10 \mathrm{~dB}$. The results are presented in Fig. 9. The residual linear distortions can be quantified by computing the gap to the AWGN channel by means of the normalized $E_{\mathrm{S}} / N_{0}$, considering the 3 -dB gain of the TWTA at $10-\mathrm{dB}$ OBO. In the scenario with 27.5 Mbaud symbol rate and $20 \%$ roll-off, the joint application of the advanced channel compensation techniques reduces the gap to the AWGN channel from $1.47 \mathrm{~dB}$ to $0.58 \mathrm{~dB}$ and from $1.84 \mathrm{~dB}$ to $0.34 \mathrm{~dB}$ for 16 -APSK and 32-APSK, respectively, as compared to static data pre-distortion and linear equalization. Here, successive data pre-distortion alone only provides gains of $0.24 \mathrm{~dB}$ and

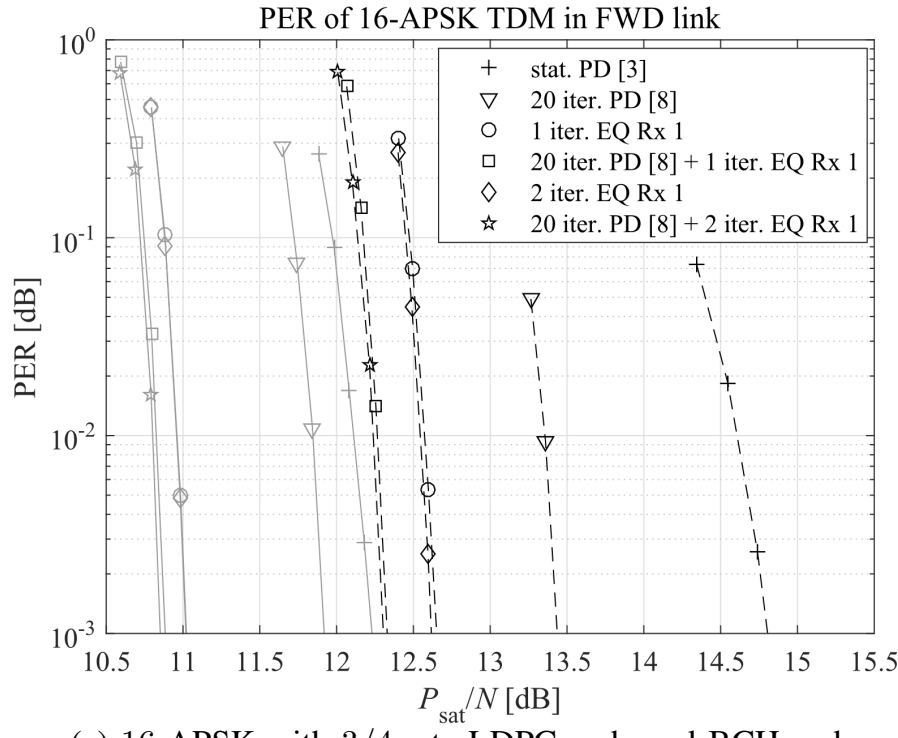

(a) 16-APSK with 3/4-rate LDPC code and BCH code.

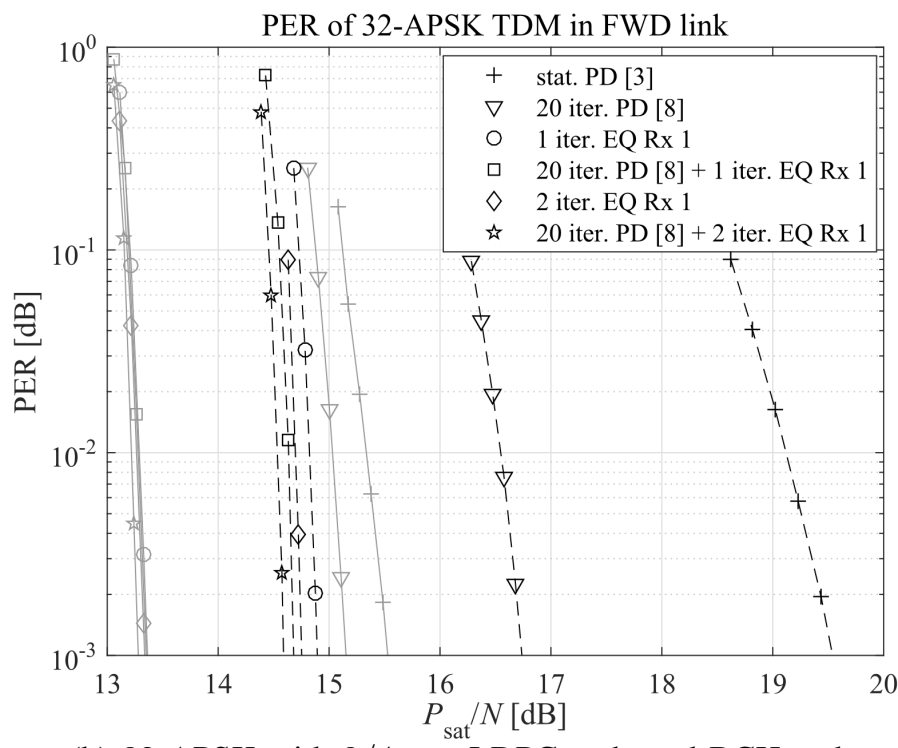

(b) 32-APSK with 3/4-rate LDPC code and BCH code.

Fig. 8: PER of single-carrier TDM with static/successive data pre-distortion at the transmitter and/or non-linear distortion cancellation at the receiver in the satellite forward link at practical OBO. Solid lines represent the scenario with $20 \%$ roll-off and 27.5 Mbaud symbol rate, while the dashed lines correspond to the scenario with $5 \%$ roll-off and 34 Mbaud symbol rate.

$0.33 \mathrm{~dB}$, while iterative equalization reduces the gap by 0.68 $\mathrm{dB}$ and $1.33 \mathrm{~dB}$. In the scenario with 34 Mbaud symbol rate and $5 \%$ roll-off, the gap to the AWGN channel is reduced from $1.87 \mathrm{~dB}$ to $0.74 \mathrm{~dB}$ and from $2.55 \mathrm{~dB}$ to $0.48 \mathrm{~dB}$ for 16-APSK and 32-APSK, respectively. In this case, successive data pre-distortion alone only provides gains of $0.78 \mathrm{~dB}$ and $1.39 \mathrm{~dB}$, while iterative equalization reduces the gap by 1.07 $\mathrm{dB}$ and $2 \mathrm{~dB}$. These simulations confirm that the proposed equalizer better compensates the linear distortions. In addition, due to the respectively larger gains achieved in the non-linear 


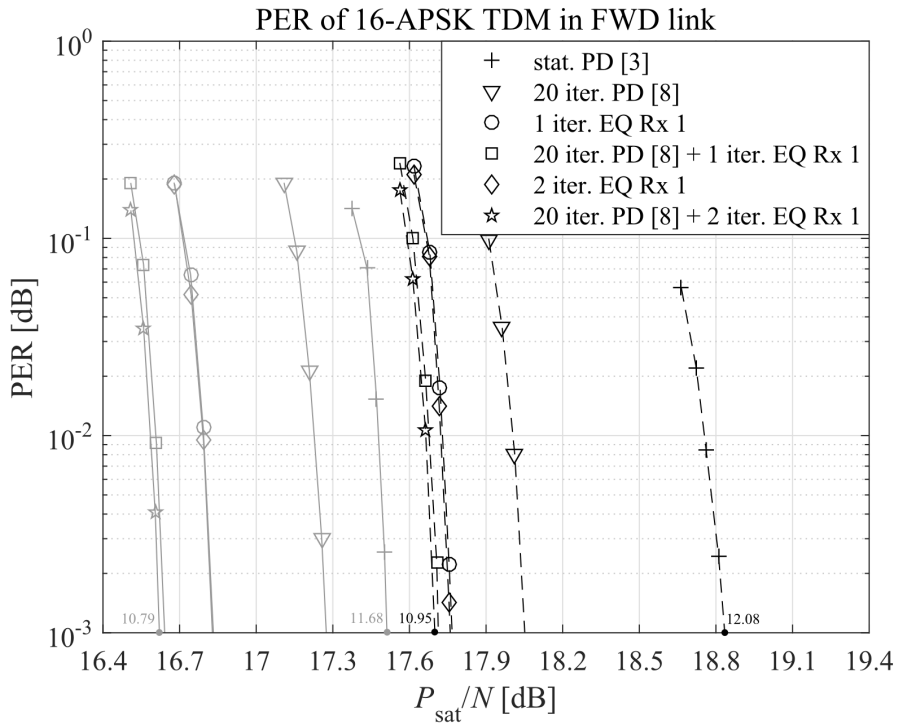

(a) 16-APSK with 3/4-rate LDPC code and BCH code.

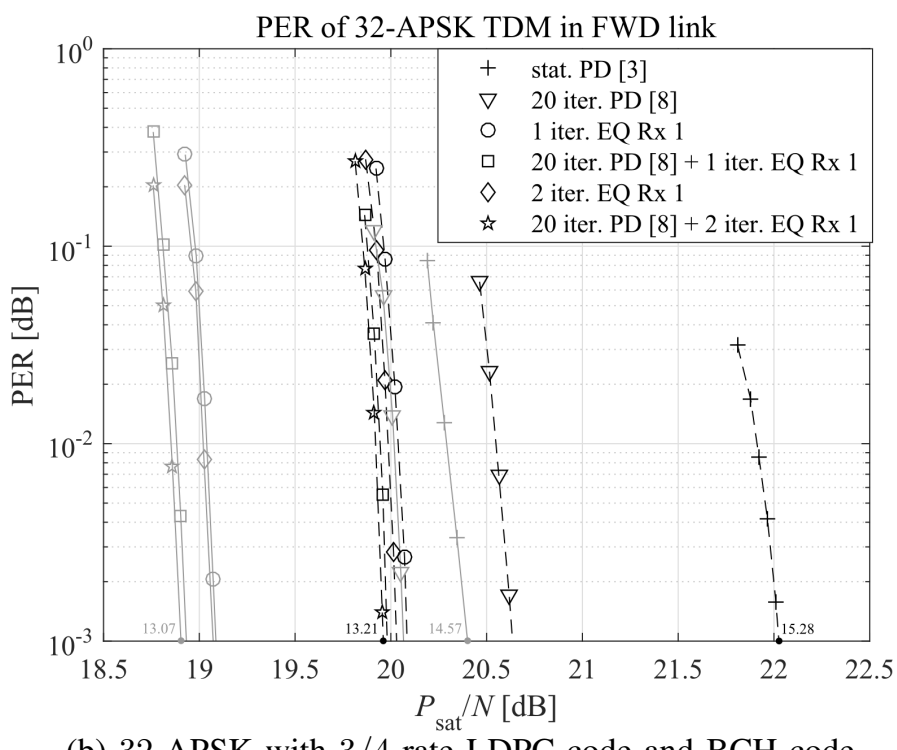

(b) 32-APSK with 3/4-rate LDPC code and BCH code.

Fig. 9: PER of single-carrier TDM with static/successive data pre-distortion at the transmitter and/or non-linear distortion cancellation at the receiver in the satellite forward link at high OBO of $10 \mathrm{~dB}$. Solid lines represent the scenario with $20 \%$ roll-off and 27.5 Mbaud symbol rate, while the dashed lines correspond to the scenario with $5 \%$ roll-off and 34 Mbaud symbol rate. Normalized $E_{\mathrm{s}} / N_{0}$ is presented at selected points.

channel shown in TABLE I, it can be argued that the proposed equalizer better compensates also the non-linear distortions as compared to the pre-distortion solution.

The presented considerable improvements in energy efficiency enable the use of higher order modulation together with very high symbol rates close to the limits of the transponder bandwidth, as well as very low roll-off factors. As a result, the spectral efficiency of the air interface is increased, which translates into increased user data rates and satellite system capacity.

\section{Complexity}

The complexity of the proposed equalizer is evaluated as the number of complex-valued multiplications required for the processing of one information symbol, since the complexity is linear with the number of symbols. The non-linear equalizer can apply the characteristics of the IMUX/OMUX filters and the TWTA to estimate the distorted received symbols, as shown in Fig. 5. In this case, the complexity of the processing blocks can be analyzed as follows:

- The two SRRCFs are applied to an oversampled version of the signal. Therefore, pulse shaping and matched filtering require $2 \eta N_{\mathrm{SRRCF}}$ complex-valued multiplications, where $\eta$ is the oversampling factor and $N_{\mathrm{SRRCF}}$ is the number of symbol durations in the SRRCF impulse response length.

- The IMUX and OMUX IIR filters are also applied with oversampling. As a result, the required operations are $\eta\left(2 N_{\text {IMUX }}+1\right)+\eta\left(2 N_{\text {OMUX }}+1\right)$, where $N_{\text {IMUX }}$ and $N_{\text {OMUX }}$ are the filter orders.

- The TWTA transfer function can be applied by means of interpolation or extrapolation with the oversampled signal. This only requires 2 real-valued multiplications per amplitude or phase components, as well as 3 real-valued multiplications per sample for the complex-polar and polar-complex transformations, which is approximately equivalent to 1 complex-valued multiplication per sample, i.e., $\eta$ operations per symbol.

- The linear equalizer is applied at twice the symbol rate, and it requires 42 complex-valued multiplications.

- The ML demodulator operates at the symbol rate, and evaluates the Euclidean distances to the reference constellation points. This can be efficiently implemented by means of $3 M$ table look-ups and $3 M$ real-valued additions, and as a result has a negligible complexity.

- Finally, the scaling of the symbols by the respective attenuation factors requires 1 operation per symbol.

The operations are repeated over $N_{i}$ iterations. The complexity can be summarized as:

$$
\begin{aligned}
C_{\mathrm{EQ}}= & N_{i}\left[\eta\left(2 N_{\mathrm{SRRCF}}+2 N_{\mathrm{IMUX}}+2 N_{\mathrm{OMUX}}+3\right)+\right. \\
& \left.+43+C_{\mathrm{PD}}\right]
\end{aligned}
$$

where $C_{\mathrm{PD}}$ is the complexity of the pre-distortion scheme. It has been shown that even 1 iteration is sufficient to provide the majority of the gain. Therefore, the complexity can be evaluated to $C_{\mathrm{EQ}}=771+C_{\mathrm{PD}}$ for $\eta=8, N_{\mathrm{SRRCF}}=32$, $N_{\text {IMUX }}=7, N_{\text {OMUX }}=5$ and $N_{i}=1$.

The proposed equalizer can also apply a memory polynomial model to estimate the distorted received symbols as shown in Fig. 5. According to [6], a memory polynomial model with highest non-linear order $P$ and memory of $Q$ has $(Q+1)(P+1) / 2$ coefficients, whereby only the odd non-linear orders are used. Since the training of the memory polynomial model can be applied sporadically to follow the slow changes in the channel, the real-time complexity only comes from the application of the model coefficients to the signal. Therefore, in this case, the complexity of the proposed equalizer can be 
expressed as:

$$
C_{\mathrm{EQ}, \text { poly }}=N_{i}\left[(Q+1)(P+1) / 2+43+C_{\mathrm{PD}}\right] .
$$

In this case, an equivalent complexity of $C_{\mathrm{EQ}, \text { poly }} \leq C_{\mathrm{EQ}}=$ $771+C_{\mathrm{PD}}$ can be achieved by the use of $Q=83$ and $P=$ 15 , providing sufficient channel tap resolution to the linear equalizer and sufficient accuracy in the channel model.

For comparison, the computational complexity of the studied data pre-distortion techniques at the transmitter is also discussed. Since training of the pre-distorter functions is performed only sporadically, it is excluded from the real-time complexity. Static data pre-distortion can be applied by means of a LUT, and its complexity can be considered negligible. The studied dynamic data pre-distortion scheme [6] requires $C_{\mathrm{PD}}=18$ operations per symbol for the application of the inverse channel model for $Q=5$ and $P=5$. The successive data pre-distortion scheme requires $C_{\mathrm{PD}}=2 \eta N_{\mathrm{SRRCF}} N_{i}$ operations [8], which for $\eta=8, N_{\mathrm{SRRCF}}=32$, and $N_{i}=20$ evaluates to $C_{\mathrm{PD}}=10240$ operations. The complexity of the pre-distortion techniques needs to be also taken into account when applied in conjunction with the proposed equalizer, as this can significantly increase the complexity of the receiver. It is shown that the proposed equalizer alone provides significant gain without pre-distortion, and even outperforms the transmitter technique. While the dynamic data pre-distortion solution is computationally efficient, it also provides less gain as compared to successive data pre-distortion. These trade-offs in terms of performance, complexity and cost are to be taken into account by satellite model manufacturers when choosing the optimum configuration of the gateway and user terminal equipment.

\section{CONCLusion}

In this paper, symbol-based equalization with non-linear distortion cancellation has been proposed as an addition to the state-of-the-art linear equalizer at the user terminal receiver in the DVB-S2X satellite forward link. The improved receiver is particularly suitable for application with the recently specified DVB-S2X reference scenarios, including wide-band carriers with low roll-off factors, high symbol baud rates, and highorder modulations. The performance of the receiver has been assessed for a single-carrier $36-\mathrm{MHz}$ transponder with higher 34 -MBaud symbol rate with lower roll-off of $5 \%$, as well as with lower 27.5-MBaud symbol rate and higher roll-off of $20 \%$, using a comprehensive model of the satellite forwardlink channel with IMUX and OMUX filter responses, nonlinear TWTA characteristics, a phase noise mask and AWGN. The use of simple ML demodulation with hard decision in the cancellation loop presented only a marginal degradation in the performance as compared to a receiver structure using soft information exchange with the LDPC decoder, and convergence of the distortion cancellation is formally shown for higher number of iterations. In addition, the improved receiver showed a superior performance in the non-linear channel with memory as compared to a number of predistortion techniques at the transmitter, including dynamic data pre-distortion, successive data pre-distortion, and standard static data pre-distortion. The joint application of successive data pre-distortion and iterative symbol-based equalization demonstrated SNR gains of up to $2.5 \mathrm{~dB}$ and $4.95 \mathrm{~dB}$ in the high-symbol-rate scenario with 16-APSK and 32-APSK, respectively. The complexity of the channel compensation techniques has been evaluated to provide a reference for satellite modem manufacturers. The study shows that significant and consistent performance gains can be obtained even after one equalization iteration, while two iterations only provide marginal further improvement. It is the objective of this paper to highlight these performance gains at low number of iterations, in order to maintain low complexity of the receiver. The results promote the proposed equalizer as an effective solution, enabling the increase of both user rates and system capacity. Future work includes a deeper study on termination criteria for the iterative equalizer, as well as the study on imperfect channel estimation and robustness of the equalizer with varying channel characteristics.

\section{REFERENCES}

[1] Second Generation Framing Structure, Channel Coding and Modulation Systems for Broadcasting, Interactive Services, News Gathering and Other Broadband Satellite Applications; Part II: S2-Extensions (DVB$S 2 X)$, Digital Video Broadcasting (DVB) Std. ETSI EN 302 307-2, Oct. 2014.

[2] "ICT-2011.1.1 BATS D4.1: Satellite Network Mission Requirements," Broadband Access via Integrated Terrestrial and Satellite Systems (BATS) European Project, Tech. Rep., 2012.

[3] Implementation Guidelines for the Second Generation System for Broadcasting, Interactive Services, News Gathering and Other Broadband Satellite Applications; Part II: S2-Extensions (DVB-S2X), Digital Video Broadcasting (DVB) Std. ETSI TR 102 376-2, Mar. 2015.

[4] E. Casini, R. De Gaudenzi, and A. Ginesi, "DVB-S2 modem algorithms design and performance over typical satellite channels," Int. J. Satellite Commun. Netw., vol. 22, pp. 281-318, Jun. 2004.

[5] G. Karam and H. Sari, "Analysis of predistortion, equalization, and ISI cancellation techniques in digital radio systems with nonlinear transmit amplifiers," IEEE Trans. Commun., vol. 37, no. 12, pp. 1245-1253, Dec. 1989.

[6] L. Ding, G. T. Zhou, D. R. Morgan, Z. Ma, J. S. Kenney, J. Kim, and C. R. Giardina, "A robust digital baseband predistorter constructed using memory polynomials," IEEE Trans. Commun., vol. 52, no. 1, pp. 159-165, Jan. 2004.

[7] D. Zhou and V. E. DeBrunner, "Novel adaptive nonlinear predistorters based on the direct learning algorithm," IEEE Trans. on Signal Proces., vol. 55, no. 1, pp. 120-133, Jan. 2007.

[8] B. F. Beidas, R. I. Seshadri, and N. Becker, "Multicarrier Successive Predistortion for Nonlinear Satellite Systems," IEEE Trans. Commun., vol. 63, no. 4, pp. 1373-1382, Apr. 2015.

[9] C. Yu, L. Guan, E. Zhu, and A. Zhu, "Band-limited Volterra seriesbased digital predistortion for wideband RF power amplifiers," IEEE Trans. Microw. Theory Techn., vol. 60, no. 12, pp. 4198-4208, Dec. 2012.

[10] N. Kelly, M. Allegue-Martinez, P.-D. Arapoglou, and A. Zhu, "Bandwidth-constrained digital pre-distortion technique for multi-carrier satellite communications," Int. J. Satellite Commun. Netw., Apr. 2015.

[11] G. Karam and H. Sari, "A data pre-distortion technique with memory for QAM radio systems," IEEE Trans. Commun., vol. 39, no. 2, pp. 336-344, Aug. 1991.

[12] J. Proakis, Digital Communications, 4th ed. McGraw Hill, Aug. 2000

[13] D. Ampeliotis, A. A. Rontogiannis, K. Berberidis, M. Papaleo, and G. E. Corazza, "Turbo equalization of non-linear satellite channels using soft interference cancellation," in Proc. 4th Advanced Satellite Mobile Systems Conference (ASMS2008), Bologna, Italy, Aug. 26-28 2008, pp. 289-292.

[14] S. Dimitrov, "Iterative cancellation of non-linear distortion noise in digital communication systems," IEEE Trans. Commun., vol. 63, no. 6, pp. 2325-2336, Jun. 2015. 
[15] _ , "Non-linear distortion noise cancellation for satellite return links," in Proc. IEEE International Conference on Communications (IEEE ICC 2016), Kuala Lumpur, Malaysia, May 23-27 2016.

[16] Second Generation DVB Interactive Satellite System (DVB-RCS2); Part 2: Lower Layers for Satellite Standard, Digital Video Broadcasting (DVB) Std. ETSI EN 301 545-2, Jan. 2012.

[17] S. Dimitrov, "Non-linear distortion noise cancellation for satellite forward links," in Proc. 8th Advanced Satellite Multimedia Systems Conference (ASMS2016), Palma de Mallorca, Spain, Sep. 5-7 2016.

[18] E. Biglieri, S. Barberis, and M. Catena, "Analysis and compensation of nonlinearities in digital transmission systems," IEEE J. Sel. Areas Commun., vol. 6, no. 1, pp. 42-51, Jan. 1988.

[19] J. Kim and K. Konstantinou, "Digital predistortion of wideband signals based on power amplifier model with memory," Electron. Lett., vol. 37, pp. 1417-1418, Nov. 2001.

[20] H. Chen and A. M. Haimovich, "Iterative estimation and cancellation of clipping noise for OFDM signals," IEEE Commun. Lett., vol. 7, no. 7, pp. 305-307, Jul. 2003.

[21] Second Generation Framing Structure, Channel Coding and Modulation Systems for Broadcasting, Interactive Services, News Gathering and Other Broadband Satellite Applications (DVB-S2), Digital Video Broadcasting (DVB) Std. ETSI EN 302 307, Aug. 2009.

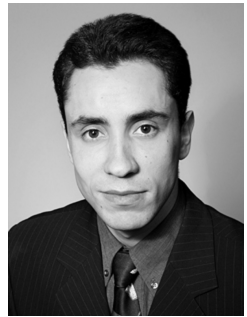

Svilen Dimitrov (S'09-M'13) received the BSc degree in electrical engineering and computer science in 2008, and the MSc degree in communications, systems and electronics in 2009 from Jacobs University Bremen, Germany. He obtained his $\mathrm{PhD}$ degree in electrical engineering in 2012 from the University of Edinburgh, UK. He wrote his BSc thesis, MSc thesis and $\mathrm{PhD}$ thesis in collaboration with Airbus Germany, EADS Germany, and EADS UK. Work included the modeling of the optical wireless channel in an aircraft cabin through Monte Carlo ray-tracing techniques and maximization of throughput of digital modulation schemes for optical wireless communications with non-linear distortion. From 2013, he is appointed as a researcher and project manager at the German Aerospace Center (DLR). His main interests are the research and development of next generation communication systems, including satellite communications, terrestrial mobile communications and optical wireless communications ( $\mathrm{Li}-\mathrm{Fi}$ ), computer-aided design, test and optimization, channel modelling, modulation techniques, air interface design, interference and resource management. $\mathrm{He}$ has published articles in highly renowned journals, such as IEEE JSAC, IEEE TCOM, IEEE TWC, IEEE/OSA JLT, IJSCN, and conferences, such as IEEE GLOBECOM, IEEE ICC, IEEE VTC, EuCAP, EuCNC, ASMS, ICSOS and $\mathrm{Ka}$ Conference. He is co-author of the book "Principles of LED Light Communications: Towards Networked Li-Fi" by Cambridge University Press. 